\title{
AMR Proximity Sensor with Inherent Demodulation
}

\author{
Pavel Ripka, Jan Vyhnanek, Michal Janosek and Jan Vcelak
}

\begin{abstract}
Our novel position sensor is based on the combination of the eddy-current and permeability effects. The primary field is excited by a coil, but instead of induction coil, the sensing part uses anisotropic magnetoresistor (AMR) which also measures DC magnetic field. As the AMR is being flipped at the excitation frequency, the sensor is self-demodulated and the output is DC. The AMR sensitivity does not depend on frequency; therefore this sensor can be used at ultralow frequencies, where coils fail as sensors. We show the response of our sensor to ferromagnetic and non-ferromagnetic metals and possibilities to distinguish between them. We also show that our sensor can measure position through the conducting sheath.
\end{abstract}

Index Terms-AMR sensor, magnetoresistor, position sensor.

\section{INTRODUCTION}

$\mathrm{T}$ WO families of proximity detectors and distance sensors are based on magnetic principle:

1. DC magnetic sensors mostly use permanent magnet as a source of the field. The magnet may be attached to the target or the sensor. In the second case the target is ferromagnetic and changes the field shape and its amplitude in the sensor location. 2. Eddy-current proximity detectors and distance sensors are most often based on the change of the quality factor of the coil caused by eddy currents in the target from conducting material $[1,2]$. These sensors are often referred as Inductive Sensors. Our sensor belongs primarily to the second group, but it is also able to measure DC response. The difference is that the source of the DC field is a coil instead of permanent magnet.

\section{A. Inductive sensors}

Inductive sensors usually work in the frequency range of $1 \mathrm{kHz}$ to $100 \mathrm{kHz}$. New trends of these sensors are described in [3]. These sensors have been also miniaturized by using printed coils [4] or CMOS technology [5]. At high frequencies the penetration depth is very small and the sensor is also strongly influenced by parasitic capacitances. At low frequencies the sensitivity of such sensor is small, which is a consequence of the induction law: the induced voltage is proportional to

Submitted for publication March 17, 2014. This work has been supported by the Grant Agency of the Czech Republic (GACR) under the grant P102/12/2177.

P. Ripka is with the Faculty of Electrical Engineering and University Centre of Energy Efficient Buildings, Czech Technical University, 16627 Prague, Czech Republic (e-mail: ripka@fel.cvut.cz).

J. Vyhnanek is with the Faculty of Electrical Engineering and University Centre of Energy Efficient Buildings, Czech Technical University, 16627 Prague, Czech Republic (e-mail: vyhnajan@fel.cvut.cz). frequency. However, for some applications it is desirable to use very low working frequency. At low frequency and low permeability the penetration depth is high and the sensor can be used to measure a position of ferromagnetic target through a conducting sheath. The requirement is that the sheath has low permeability - if it is from ferrous material, its permeability can be lowered by DC saturation.

\section{B. AMR position sensor}

Our novel solution separates the transmission coil from the magnetic field sensor, which is in our case Anisotropic magnetoresistor (AMR) with a frequency response starting from DC [6]. AMR sensors are based on a thin film Permalloy strip, which forms a single domain. The external magnetic field rotates the permanent magnetization of the domain and causes a change of its electrical conductivity. Large external field can cause that the single-domain state is damaged, which results in hysteresis and drop of sensitivity. The cure is called flipping: short periodical pulses of magnetic field which remagnetize the sensor core and restore its single-domain state. If the flipping pulses are bipolar, the sensor output also periodically reverts its polarity, i.e. it is modulated by the flipping frequency. Flipping brings also other advantages:

1. suppression of the sensor offset

2. suppression of the crossfield effect [7]

3. doubling the sensitivity

Anisotropic magnetoresistors were already used in eddycurrent NDT with high spatial resolution [8]. Compared to induction coil, AMR sensors have smaller size and their response is to some limit frequency independent. Therefore AMR sensors can be used at much lower frequencies than induction coils, which have sensitivity proportional to frequency. Fig. 1 shows the conventional system using AMR: $f_{\text {flip }}$ is typically $1 \mathrm{~Hz}$ to $1 \mathrm{kHz}$, the AMR output is $\mathrm{AC}$ at $f_{\text {exc }} \pm$ $f_{\text {flip }}$ frequency. Synchronous demodulator at its output is controlled by $f_{\text {flip }}$ and the dominant signal at its output is at $f_{e x c}$.

M. Janosek is with the Faculty of Electrical Engineering, Czech Technical University, 16627 Prague, Czech Republic (e-mail: janosem@fel.cvut.cz).

J. Vcelak is with the Centre of Energy Efficient Buildings, Czech Technical University, 16627 Prague, Czech Republic (e-mail: vcelakj1@,fel.cvut.cz).

Copyright (c) 2013 IEEE. Personal use of this material is permitted. However, permission to use this material for any other purposes must be obtained from the IEEE by sending a request to pubs-permissions@ieee.org. 


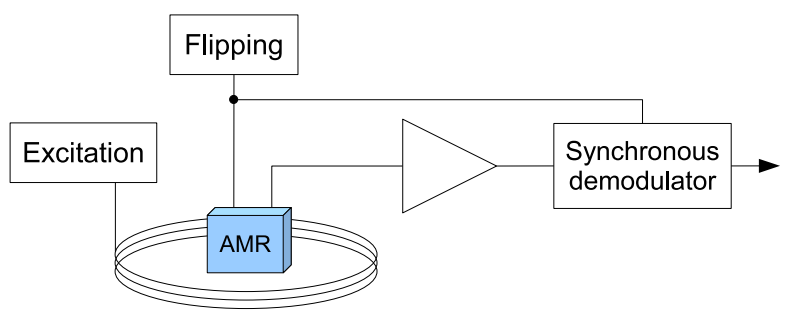

Figure 1. Flipped AMR with external demodulator. The sensor output is $\mathrm{AC}$ at $f e x c$

\section{Self-demodulation}

Our new concept of self-demodulated sensor is shown in Fig. 2. The excitation field is a squarewave of the same frequency as the flipping pulses, so that the sensor output is at DC (and also at $\left.2 f_{\text {exc }}\right)$.

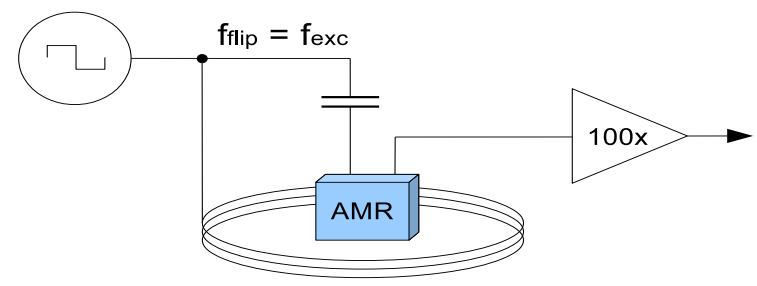

Figure 2. AMR with inherent demodulation. The primary sensor output is DC.

The possibility to use flipping for signal demodulation was predicted by Tumanski [9], but to our knowledge we were the first who experimentally proved this concept and used it for sensing. The output signal is DC voltage without any external demodulator. We can use the same sensor to separately measure DC magnetic response and eddy-current response.

Our method has three advantages over DC magnetic position sensors:

1. it does not require any permanent magnet,

2. it suppresses the influence of the external DC magnetic fields and magnetic remanence of iron parts, and

3 . it works also for non-magnetic conducting targets.

The only limitation is the measuring range of the AMR sensor as the DC magnetic field results in the AC output signal. However, using an appropriate processing and feedback compensation the DC magnetic field can be suppressed. By this sensor we can separately measure DC magnetic response and eddy-current response in order to compensate for the target temperature-dependent permeability.

\section{THE MEASUREMENTS}

The prototype sensor is shown in Fig. 3

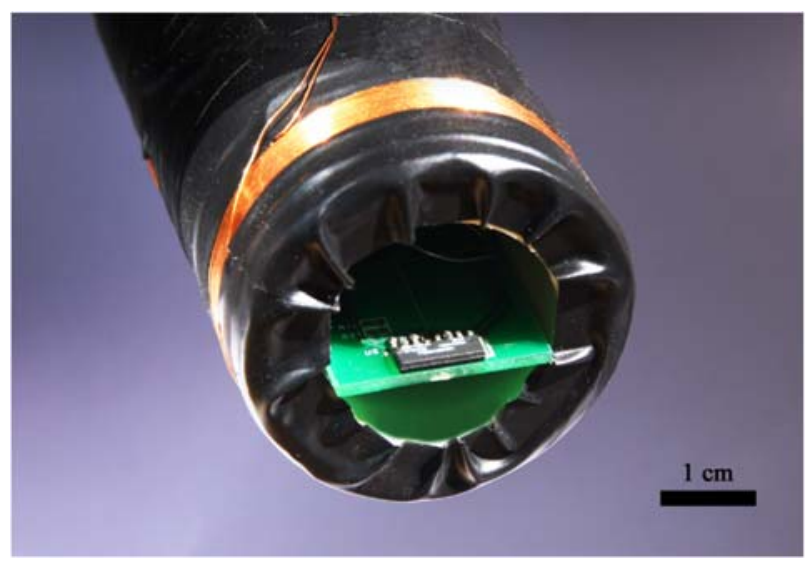

Figure 3. Experimental proximity sensor with the AMR inside the excitation coil

It uses single Honeywell HMC 1001 AMR sensor and AD8429 instrumentation amplifier with the gain of 100 . The sensor is inside the $46 \mathrm{~mm}$ diameter, $22 \mathrm{~mm}$ long excitation solenoid powered by $70 \mathrm{~mA}$ p-p squarewave current, which generates $115 \mathrm{~A} / \mathrm{m}$ field at the end of the coil and $12 \mathrm{~A} / \mathrm{m}$ field in $4 \mathrm{~cm}$ distance. The sensor is located in the coil axis at the coil end. Flipping is made using the integrated flipping coil by $1.2 \mathrm{~A}$ p-p current created by discharging a $6.8 \mathrm{nF}$ capacitor directly coupled to the $30 \mathrm{~V}$ p-p square-wave excitation waveform from $50 \Omega$ generator. Fig. 4 shows the typical response of the selfdemodulated sensor to conducting target for $1 \mathrm{kHz}$ excitation frequency. Basic DC shift is caused by the fact that the sensor is subjected to the full excitation field which is selfdemodulated into the DC voltage. If necessary this basic DC shift can be compensated either by feedback current in the sensor or by voltage shift in the pre-amplifier. The exponential pulses are caused by eddy currents in the target. Notice that after self-demodulation all pulses have the same polarity and therefore they contribute to DC output. If the target is ferromagnetic, further DC shift is caused by its permeability. The eventual squarewave component is caused by external magnetic field or (in the case of ferromagnetic target) by the target remanence.

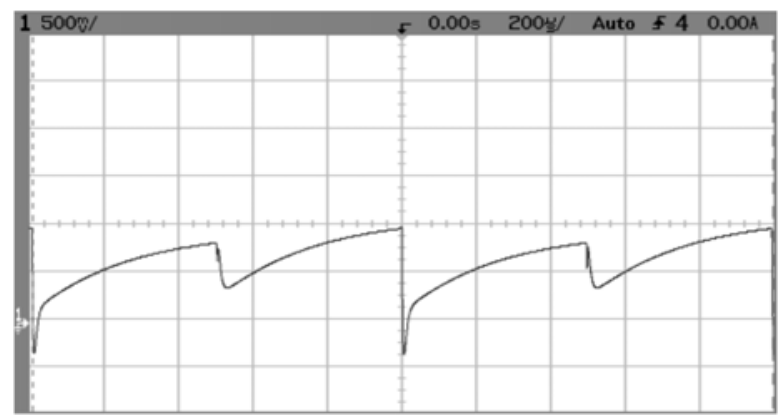

Figure 4. Sensor response to $\mathrm{Al}$ target, $f$ exc $=1 \mathrm{kHz}$

When measuring the target position through the sheath the excitation frequency should be adjusted so that the penetration depth is larger than the sheath thickness. In such case the eddy 
currents decay fast and the magnetic field propagates through the sheath towards the target. This situation is shown in Fig. 5.
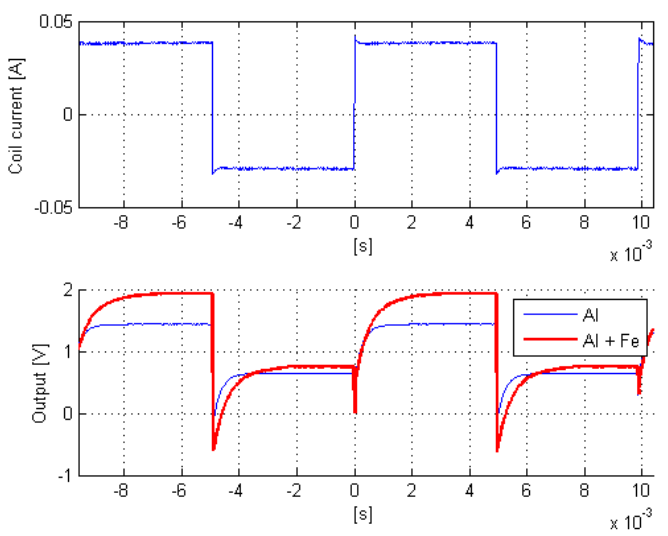

Figure 5. Excitation current (upper trace) and the sensor response (lower trace) to Al sheath alone with the thickness below the penetration depth. The eddy currents decay fast and the magnetic field propagates through the $2.5 \mathrm{~mm}$ sheath, fexc $=100 \mathrm{~Hz}$. Signal belonging to the ferrous target inside the sheath is visible.

Fig. 6 shows sensor response also to $10 \mathrm{~Hz}$ and $1 \mathrm{kHz}$ excitation current. While at $1 \mathrm{kHz}$ the sensitivity is smaller, $10 \mathrm{~Hz}$ give nice response for $\mathrm{Fe}$ target, however the transitional part is too short for time-domain analysis. $100 \mathrm{~Hz}$ excitation was therefore selected as optimum for this configuration.
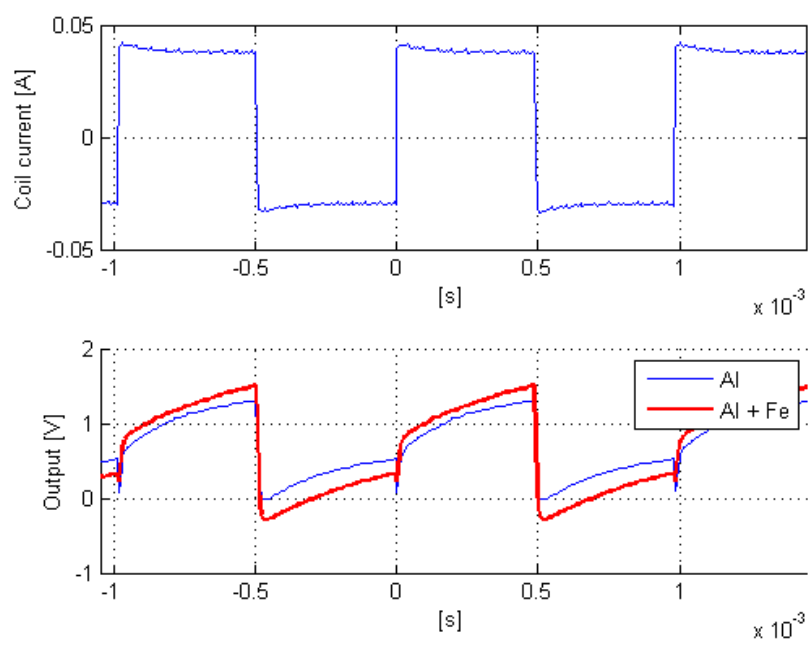

a)

$1 \mathrm{kHz}$
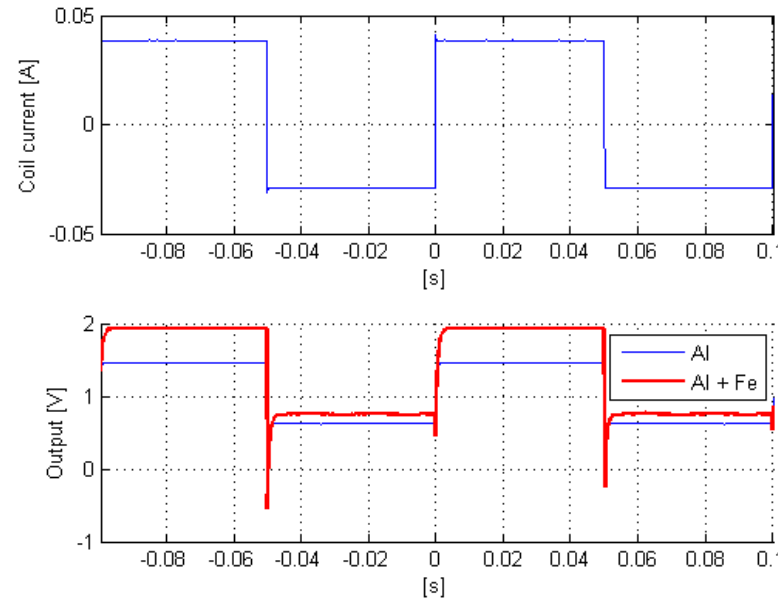

b)

$10 \mathrm{~Hz}$

Figure 6. Excitation current and sensor output for a) $1 \mathrm{kHz}$ and b) $10 \mathrm{~Hz}$. The sheath and target are the same as in Fig.5.

Fig. 7 shows the calibration of the selected setup. We measured a DC response from both ferromagnetic and aluminum targets through the same $2.5 \mathrm{~mm}$ aluminum sheath. The target distance is measured from the end of the excitation coil. The frequency dependence in this case is caused not only by the eddy currents in the sheath, but also in the target. Conventional induction coil at this frequency shows very small sensitivity and cannot be used.

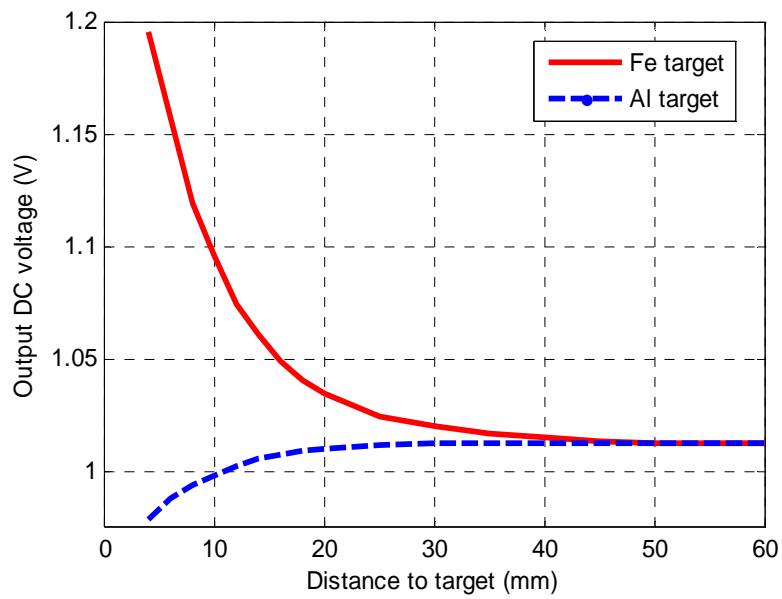

Figure 7. DC output of self-demodulating AMR proximity detector sensing iron and $\mathrm{Al}$ target through $2.5 \mathrm{~mm} \mathrm{Al}$ sheath. fexc $=100 \mathrm{~Hz}$

\section{PROCESSING OF THE AC SIGNAL}

The used principle is very flexible and it allows estimating the target properties using techniques developed for eddy-current non-destructive testing and mine detection. The analysis can be performed both in the frequency and time domain. 


\section{A. Processing in the frequency domain}

Sinewave excitation field at multiple frequencies is ideal for analysis in the frequency domain. For position sensor the overall circuit simplicity is an important aspect, which is a strong argument for using squarewave excitation. However, the signal spectrum for squarewave excitation is rather complex as illustrated in Fig. 8. The $100 \mathrm{~Hz} \pm 100 \mathrm{~Hz}$ signal created by mixing $100 \mathrm{~Hz}$ excitation with $100 \mathrm{~Hz}$ flipping frequencies has two products: $\mathrm{DC}$ which is the main sensor output and signal at $200 \mathrm{~Hz}$. However, part of the $200 \mathrm{~Hz}$ signal comes from the third harmonic component of the excitation squarewave mixed with flipping $(200=300-100)$. The $100 \mathrm{~Hz}$ spectrum component is caused by DC signal (including the sensor offset) modulated by $100 \mathrm{~Hz}$. A small part of this signal can be also caused by capacitive or inductive feedthrough from the excitation. In general the higher even harmonics are caused by squarewave spectrum components modulated by $100 \mathrm{~Hz}$ signal and the higher odd harmonics are caused by AMR sensor nonlinearities.

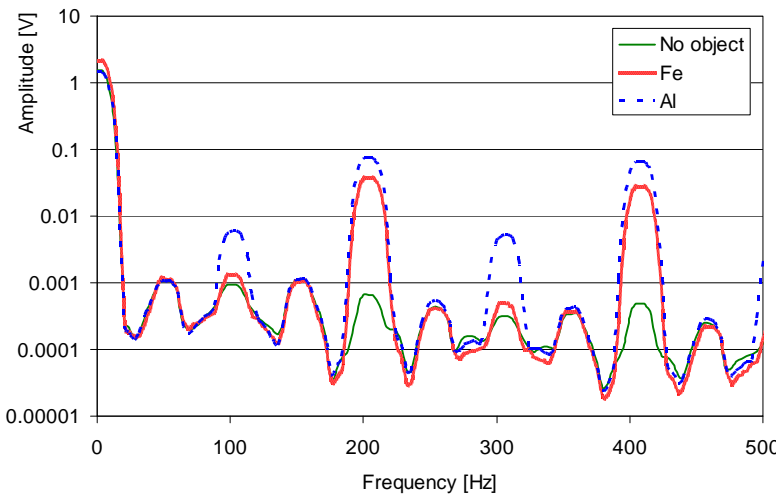

Figure 8. Spectrum of sensor output for various targets, $100 \mathrm{~Hz}$ excitation, $100 \mathrm{~Hz}$ flipping.

\section{B. DC field compensation}

We have shown that the external DC field together with AMR non-linearity creates harmonics. More serious is the danger of the saturation at larger fields. Therefore we decided to employ DC field compensation. In our case the frequency band of the feedback loop is limited to $5 \mathrm{~Hz}$, which lefts the $100 \mathrm{~Hz}$ field unaffected. The effect of the feedback compensation on output spectrum is shown in Fig. 9. In this case the sensor is placed inside the magnetic shielding, so that the DC component is caused only by the sensor offset. An unwanted consequence of the feedback compensation is the increased noise in sidebands of $100 \mathrm{~Hz}$ and all odd harmonics.

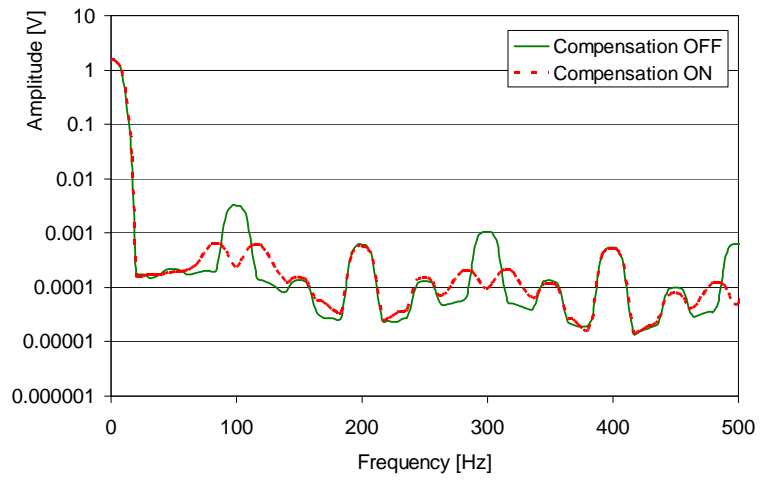

Figure 9. Spectrum of sensor output with feedback compensation on/off in magnetic shielding

When the sensor is exposed to the Earth's magnetic field of 50 $\mu \mathrm{T}$ (Fig. 10) the amplitude of $100 \mathrm{~Hz}$ and odd harmonic components is significantly higher, confirming the origin of this signal. After compensation the spectrum is practically the same as in Fig. 9.

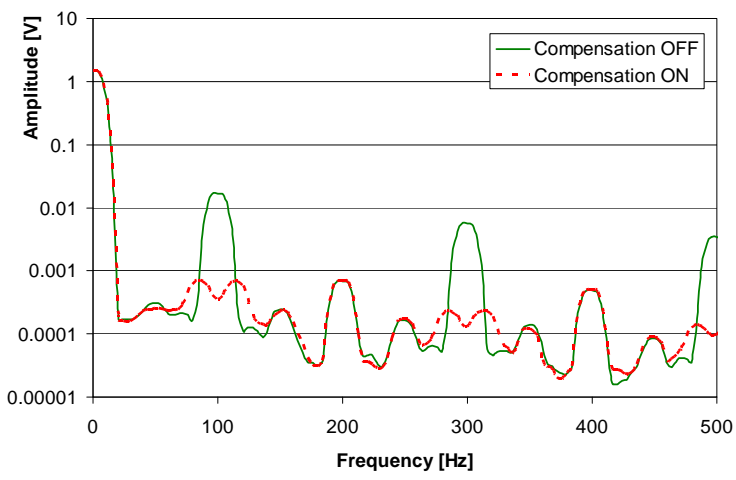

Figure 10. Spectrum of sensor output with feedback compensation on/off in the Earth's field

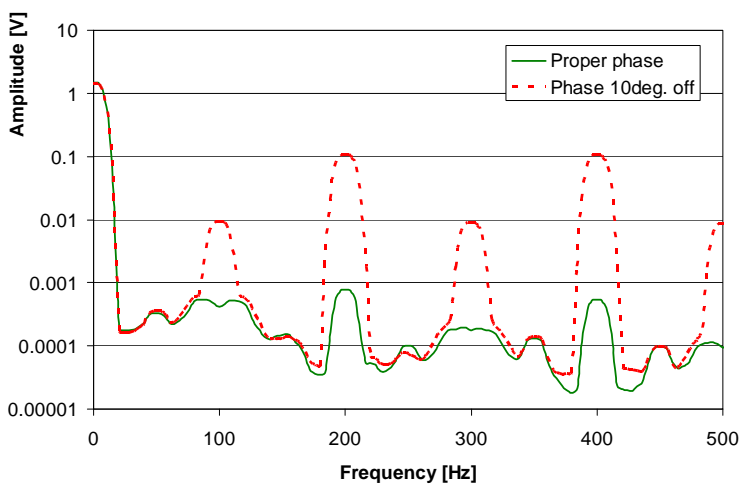

Figure 11. Spectrum of sensor output for different phase between excitation field and flipping current with active feedback compensation

DC field is not the only source of the frequency of $100 \mathrm{~Hz}$ as shows the spectrum for the aluminum target in Fig. 8. Similar 
behavior was observed when an improper phase between the excitation field and the flipping current deteriorates the rectification and results in an output waveform with negative peaks with the repetitive frequency of $200 \mathrm{~Hz}$ (Fig. 11 and 12). Deteriorated function of the feedback results in the rise of 100 $\mathrm{Hz}$ in the frequency domain. Due to its origin the remaining $200-\mathrm{Hz}$ signal cannot be eliminated by the feedback designed for the DC field compensation. At the low excitation frequency the phase accuracy is however not critical as the largest delay in our setup was the recovery time of the AMR sensor after flipping, which was only $30 \mu$ s.

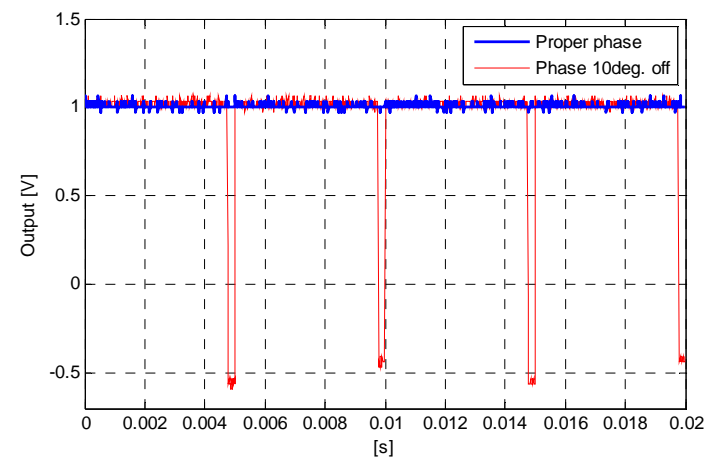

Figure 12. Sensor output for different phase in time-domain

\section{Signal processing in the Amplitude domain}

Analysis of amplitude and phase shifts of shown spectra is not an easy task. Therefore we decided for time-domain signal processing, which in low-frequency case appears to be more straightforward.

For the laboratory evaluation we developed a software application based on dividing the digitized waveform into two intervals, the first one measures eddy currents and the second one the steady field of the excitation coil (Fig. 13). The digitizer was 16 bit PCI-6221 from National Instruments, where a 10 $\mathrm{kHz}$ sampling rate proved to be sufficient for the $100 \mathrm{~Hz}$ excitation.

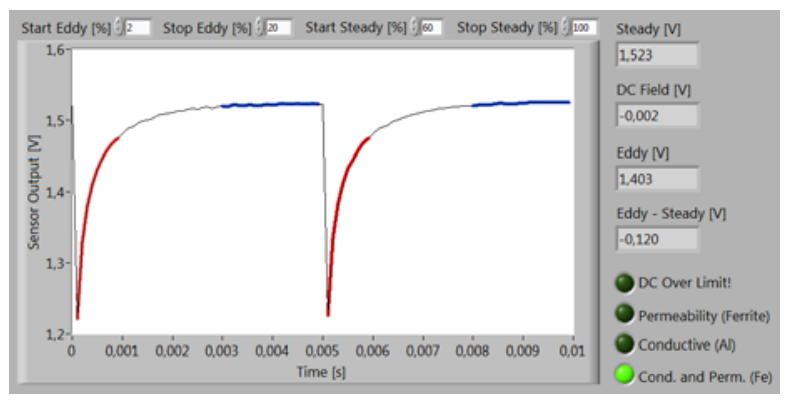

Figure 1. Sensor DC output of self-demodulating AMR proximity detector sensing iron and $\mathrm{Al}$ target through $2.5 \mathrm{~mm} \mathrm{Al}$ sheath. fexc $=100$ Hz. The "squarewave component" is compensated due to DC field feedback.

The mean value of the signal in the first interval is a function of target conductivity, permeability and distance, while the mean value in the second interval depends only on permeability and distance.
The effects of conductivity and permeability can be therefore evaluated separately using a single squarewave excitation frequency of $100 \mathrm{~Hz}$. Therefore apart from proximity detection the material type is also indicated. It should be noted, that the signal amplitude also depends on the target distance and geometry. Some apriori information about the target is therefore necessary. More information can be brought by multitone excitation, technique routinely used for mine detection.

The DC field (e.g. the Earth's field) is obtained as the squarewave component amplitude of the self-demodulated output and compensated by an external coil to suppress the nonlinearity of AMR output characteristic. Thus the linear output behavior is ensured for $\pm 700 \mathrm{~A} / \mathrm{m}$ DC field range. However the sensor frequency response, which is already low due to the low excitation frequency, is further restricted by the feedback compensation.

\section{CONCLUSIONS}

We have shown that the proposed AMR eddy current sensor can be used at very low excitation frequency $(1$ to $100 \mathrm{~Hz})$ to detect the position of ferromagnetic or massive conducting object covered by a metal sheath. For the first time we demonstrated the self-demodulation ability when the excitation and flipping signals have the same frequency. We have proven $40 \mathrm{~mm}$ detection distance for ferrous target measured through the 2.5 $\mathrm{mm}$ thick aluminum sheath. Using the time-domain signal analysis, it is also possible to identify the target material. The sensor also measures and simultaneously compensates the DC field component. This makes the sensor immune against external fields without shielding, which was used for this purpose in [10] and [11]. The present sensor is not differential, DC field compensation is made electronically and no suppression of the excitation field is necessary. Using gradiometric configuration for larger detection distances is also possible; the detection distance limit can be increased also by increasing of the excitation coil diameter. Final version of the intelligent position sensor should have electronics integrated into the sensor body, which will be the next step of the development.

\section{ACKNOWLEDGMENT}

This work has been supported by the European Union, OP RDI project No. CZ.1.05/2.1.00/03.0091 - University Centre for Energy Efficient Buildings, and by the Grant Agency of the Czech Republic (GACR) under the grant P102/12/2177.

\section{REFERENCES}

[1] T. Reininger, F. Welker, M. von Zeppelin, "Sensors in position control applications for industrial automation," Sens. Act. A, vol. 129, pp. 270274. May 2006.

[2] M. Jagiella, S. Fericean, A. Dorneich, "Progress and recent realizations of miniaturized inductive proximity sensors for automation," IEEE Sens. J., vol. 6, pp. 1734-1741, Dec. 2006.

[3] S. Fericean, R. Droxler, "New Noncontacting Inductive Analog Proximity and Inductive Linear Displacement Sensors for Industrial Automation," IEEE Sens J., vol. 7, pp. 1538-1545, Nov. 2007.

[4] H. Bartsch, T. Geiling, J. Mueller, "A LTCC low-loss inductive proximity sensor for harsh environments," Sens. Act. A, vol. 175, pp. 28-34, Mar. 2012 . 
This is the author's version of an article that has been published in this journal. Changes were made to this version by the publisher prior to publication.

The final version of record is available at http://dx.doi.org/10.1109/JSEN.2014.2325406

[5] P. H. Lo, S. H. Tseng, J. H. Yeh, et al., "Development of a proximity sensor with vertically monolithic integrated inductive and capacitive sensing units," J. of Micromech. and Microeng, vol. 23, Article Number: 035013, 2013.

[6] P. Ripka, M. Janosek, “Advances in Magnetic Field Sensors,” IEEE Sens. J., vol. 10 no. 6 , pp. 1108-1116, Jun. 2010.

[7] P. Ripka, M. Janosek, M. Butta, "Crossfield Sensitivity in AMR Sensors," IEEE Trans. Magn., vol. 45, no. 10, pp. 4514 - 4517, Oct. 2009.

[8] B. Marchand, J. M. Decitre, O. Casula, "Flexible and array eddy current probes for fast inspection of complex parts," in Rev. of Quant. Nondestr. Eval. (AIP), Kingston, vol. 29, 2010, pp. 385-392.
[9] S. Tumanski, Thin film magnetoresistive sensors. Bristol, UK: IOP, 2001, pp 76-81.

[10] M. Martino, A. Danisi, R. Losito, et al., "G.: Design of a linear variable differential transformer with high rejection to external interfering magnetic field," IEEE Trans. Magn., vol. 46, pp. 674 - 677, Feb. 2010.

[11] A. Masi, A. Danisi, R. Losito, et al., "Characterization of magnetic immunity of an ironless inductive position sensor, “ IEEE Sens. J., vol. 13, no. 3, pp. $941-948$, Mar. 2013 